\title{
Prévalence du pré-diabète, du syndrome métabolique, du diabète et des dyslipidémies chez 350 sujets recrutés au marché de Hédzranawoé à Lomé (Togo)
}

\author{
Kafui Codjo KOUASSI ${ }^{1,2 *}$, Soulemane PESSINABA ${ }^{3}$, Laconi KAAGA ${ }^{4}$, \\ Courdjo LAMBONI ${ }^{1}$ et Findibe DAMOROU ${ }^{3}$
}

${ }^{1}$ Faculté des Sciences (FDS), Département de Biochimie, 07 BP 7544 Lome 07-Togo.

${ }^{2}$ Centre Hospitalier Universitaire Campus-Département de Biochimie, Togo.

${ }^{3}$ Faculté des Sciences de la Santé-Centre Hospitalier Universitaire Campus-Service de Cardiologie, Togo.

${ }^{4}$ Centre Hospitalier Universitaire Campus- Service de Médecine Interne, Togo.

*Auteur correspondant ; E-mail : kafui.kouassi@gmail.com,ckouassi@univ-lome.tg; Tél. (+228) $90077357 /(+228) 97386580$

\section{RESUME}

Les sujets atteints de pré-diabète présentent un risque élevé de syndrome métabolique (SM) et de diabète. La présente étude avait pour but d'évaluer la prévalence du pré-diabète, du SM et du diabète dans une population fréquentant un marché urbain, puis de déterminer la prévalence des dyslipidémies chez les pré-diabétiques et diabétiques. Il s'est agi d'une étude transversale à visée descriptive portant sur 350 sujets âgés de 18 à 90 ans, recrutés au grand marché de Hédranawoè en 2015. La prévalence du pré-diabète a été évaluée suivant les critères de l'Organisation Mondiale de la Santé (OMS) et de l'American Diabetes Association (ADA). La prévalence du SM a été obtenue avec les critères de la Fédération Internationale du Diabète (FID). La prévalence du pré-diabète suivant le critère d'ADA était de $13,2 \%$ avec une glycémie moyenne à 5,9 $\pm 0,3 \mathrm{mmol} / \mathrm{l}$. Celle du SM était de $1,3 \%$ chez les femmes et de $0,8 \%$ chez hommes, avec celle du diabète qui était à 7,1\%. Celle des dyslipidémies variait de 32,0 à 60,0\% chez les diabétiques dont $40 \%$ n'était pas préalablement dépistés. La prévalence du diabète était plus importante que celle déterminée dans la population générale du Togo. La prévalence du syndrome métabolique mériterait d'être réévaluer en utilisant des critères de tour de taille établis pour des africains au Sud du Sahara. Les populations fréquentant les marchés urbains devraient être régulièrement sensibilisées sur les risques du pré-diabète, du diabète et des dyslipidémies.

(C) 2020 International Formulae Group. All rights reserved.

Mots clés: Hyperglycémie, urbain, adultes, hypertriglycéridémie, Togo.

\section{Prevalence of pre-diabetes, metabolic syndrome, diabetes and dyslipidemia in 350 subjects recruited at the Hédzranawoé market in Lomé (Togo)}

\begin{abstract}
People with pre-diabetes are at high risk for metabolic syndrome (MS) and diabetes. The purpose of the present study was to assess the prevalence of pre-diabetes, MS and diabetes in a population frequenting an urban market, and then to determine the prevalence of dyslipidemia in pre-diabetics and diabetics. This
\end{abstract}


was a descriptive cross-sectional study on 350 subjects aged 18 to 90 , recruited from the large market of Hédranawoè in 2015. The prevalence of pre-diabetes was assessed according to the criteria of the World Organization of Health (WHO) and the American Diabetes Association (ADA). The prevalence of MS was obtained using the criteria of the International Diabetes Federation (IDF). The prevalence of pre-diabetes according to the ADA criterion was $13.2 \%$ with an average blood sugar level of $5.9 \pm 0.3 \mathrm{mmol} / \mathrm{L}$. That for MS was $1.3 \%$ in women and $0.8 \%$ in men, with that of diabetes at $7.1 \%$. That of dyslipidemia ranged from 32.0 to $60.0 \%$ in diabetics. Forty percent $(40 \%)$ of these diabetics were not previously screened. The prevalence of diabetes was higher than that determined in the general population of Togo. The prevalence of metabolic syndrome should be reassessed using waist size criteria established for Africans from south of the Sahara. Populations frequenting urban markets should be regularly made aware of the risks of pre-diabetes, diabetes and dyslipidemia.

(C) 2020 International Formulae Group. All rights reserved.

Keywords: Hyperglycemia, urban, adults, hypertriglyceridemia, Togo.

\section{INTRODUCTION}

Le pré-diabète, le syndrome métabolique (SM) et le diabète sont associés à un niveau de risque croissant de maladies cardiovasculaires (Okafor, 2012). En 2017, en Afrique, 15,9 millions de personnes vivaient avec le diabète et 42,9 millions âgés de 18 à 99 ans étaient en état de pré-diabète. Le pré-diabète se caractérise par des anomalies de la glycorégulation avec une glycémie à jeun supérieure ou égale à 6,1 $\mathrm{mmol} / \mathrm{l}(110 \mathrm{mg} / \mathrm{dl})$, mais inférieure à 7,0 $\mathrm{mmol} / \mathrm{l}$ (126 mg/dl) selon l'Organisation Mondiale de la Santé (OMS). Selon l'American Association of Diabetes (ADA), il est défini par une glycémie à jeun supérieure ou égale à 5,6 mmol/l $(100 \mathrm{mg} / \mathrm{dl})$ mais inférieure à 7,0 mmol/l. Cet état de prédiabète associé à l'hypertension et à une dyslipidémie dans un contexte d'obésité abdominale entraîne le SM dont la prévalence estimée par la FID serait entre 20 à $25 \%$ des adultes du monde entier (Alberti et Zimmet, 2007). En 2010, selon l'enquête de surveillance des facteurs de risque des maladies chroniques (STEPS)-Togo de l'OMS, la prévalence de l'hyperglycémie diabétique était de 2,6\% dans les populations de 15 à 64 ans (Agoudavi et al., 2012). Ces données ne permettaient pas de connaître distinctement la prévalence du pré-diabète et du diabète dans des populations spécifiques comme celles d'un marché urbain de commerce général (Shen et al., 2016). La prévalence du SM par les critères de la FID dans cette population spécifique n’était pas non plus connue. La présente étude avait pour but d'évaluer la prévalence du pré-diabète, du SM et du diabète dans une population fréquentant un marché urbain, puis de déterminer la prévalence des dyslipidémies chez les pré-diabétiques et diabétiques.

\section{MATERIEL ET METHODES}

Il s'est agi d'une étude transversale à visée descriptive. Ont été inclus dans la présente étude, 350 sujets de 18 ans au moins, qui ont donné leur consentement oral. Les sujets exclus étaient les femmes enceintes et tous ceux dont toutes les données pertinentes pour analyses n'étaient pas fournies. Les sujets ont été recrutés entre le mardi 10 et le vendredi 13 février 2015, sur le site du marché de Hédzranawoé où les articles suivants étaient commercialisés : friperie, denrées alimentaires avec des petits restaurants, et quelques produits artisanaux. Cette étude était réalisée dans le cadre d'une enquête initiée par le Programme National de Lutte contre les Maladies Cardio-Vasculaire (PNLMCV). Elle a été réalisée par une équipe composée d'un professeur titulaire en cardiologie, de trois (03) médecins cardiologues, d'un (01) biologiste spécialisé en biochimie-nutrition, de seize (16) infirmiers diplômés d'état et de huit (08) techniciens supérieurs de laboratoire de biologie médicale. Ils ont bénéficié d'une formation pré-enquête. Les enquêteurs ont bénéficié d'un rappel sur les bonnes pratiques 
de prise du poids, de la taille, du périmètre abdominal, de la pression artérielle et sur les exigences normatives de réalisation des analyses biochimiques. Les données étaient recueillies par un questionnaire rédigé en français et renseigné par un des infirmiers recrutés. Quatre équipes ont été formées pour travailler chaque jour pendant quatre jours. La première équipe était chargée de la sensibilisation sur la prévention et les facteurs de risque cardio-vasculaire. La deuxième équipe était chargée de l'interrogatoire, la troisième des mesures physiques et anthropométriques et la quatrième des mesures biologiques.

Les autorités du marché de Hédzranawoè ont accepté la réalisation de cette enquête sur leur site, par le PNLMCV, avant l'enregistrement des 509 sujets qui s'étaient volontairement rendus dans les installations aménagées sur le site du marché. L'étude a concerné les commerçants, le personnel administratif travaillant sur le site et tous ceux qui étaient venus sur le site les 3 premiers jours du dépistage. Ainsi, la population présente sur le site du marché le mardi, le mercredi, le jeudi et le vendredi a été sensibilisée par le biais de la radio du marché sur l'objectif de l'enquête et les étapes de son déroulement. Avant chaque séance de dépistage, la population a bénéficié d'une sensibilisation sur la prévention des maladies cardio-vasculaires. Les sujets étaient recrutés les 10,11 et 12 février avec les prélèvements réalisés les 11,12 et 13 sur rendez-vous entre $8 \mathrm{~h} 00$ et $10 \mathrm{~h} 30$ minutes. Les explications étaient données la veille, sur les conditions pré-analytiques à respecter avant les prélèvements. Les sujets perdus de vue étaient attendus le 13 février 2015 après les avoir appelés. Tous les sujets disposaient d'une fiche avec les contacts du biologiste et d'un médecin pour le retrait des résultats, surtout dans les cas des perdus de vue récupérés le vendredi 13 février 2020. Au total, 159 sujets n'étaient pas revenus pour les prélèvements sanguins ou étaient revenus mais sans être dans les conditions adéquates pour les prélèvements. La fiche $\mathrm{du}$ questionnaire avait également servi à recueillir les résultats des mesures physiques, anthropométriques et biologiques.

\section{Mesures physiques et anthropométriques}

La pression artérielle a été prise à l'aide d'un tensiomètre électronique de marque OMRON (Omuron Kabushiki-gaisha Corporation du Japon) validé par l'OMS. La mesure a été effectuée chez un sujet en position assise, aux deux bras après un repos de 10 à $15 \mathrm{~min}$. Trois mesures consécutives du poids ont été effectuées chez un sujet légèrement vêtu à l'aide d'une pèse personne de marque SECA (Gmbh, Allemagne) avec une précision de $0,5 \mathrm{~kg}$. Cette mesure n'a pu être réalisée qu'auprès de 294 sujets parmi les 350 enregistrés. La taille a été mesurée sur un sujet debout à l'aide d'une toise de précision $1 \mathrm{~cm}$. Le périmètre abdominal a été mesuré sur un sujet debout, au niveau de l'ombilic à l'aide d'un mètre ruban à une précision de 1 $\mathrm{cm}$.

\section{Examens biochimiques}

Les examens biochimiques ont été effectués sur des sujets à jeun de 12 heures. Ils ont fait l'objet d'un prélèvement sanguin introduit dans un tube sec et dans un tube avec anticoagulant «Ethylène-DiamineTétra-Acétique » (EDTA). Les paramètres biochimiques évalués étaient la glycémie (GLY), la cholestérolémie totale (CT), la triglycéridémie (TG) et la fraction High Density Lipoprotein-Cholestérolémie (HDLC). Ces examens ont été réalisés sur l'analyseur de chimie sèche REFLOTRON PLUS (Roche Diagnostic, Allemagne). Les valeurs de référence utilisées pour le bilan lipidique étaient celles du NCEP (National Cholesterol Education Program) des EtatsUnis (National Institutes of Health, 2001). Le principe de mesure était basé sur la réflectance sur la bandelette-test à l'aide de la sphère intégrante d'Ulbricht. Les méthodes biochimiques utilisées étaient du mode Trinder $\left(\mathrm{H}_{2} \mathrm{O}_{2}\right.$ - Péroxydase). L'HDL-C a été obtenue par dosage plasmatique dans le surnageant après précipitation des 
chylomicrons et les fractions Low Density Lipoprotein (LDL)/Very Low Density Lipoprotein (VLDL)-cholestérol par l'action du sulfate de dextran et de l'ion $\mathrm{Mg}^{2+}$ contenus dans la bandelette réactive. $\mathrm{La}$ fraction HDL-C restée dans l'échantillon était mesurée par le même principe de mesure $\mathrm{du}$ cholestérol. Ces mesures étaient standardisées grâce à une courbe d'étalonnage de référence définie pour chaque lot à l'aide de la méthode: Glucoquant (hexokinase) pour la glycémie ; Cholesterol Oxydase p-Aminophénazone (CHOD-PAP) pour le cholesterol ; Glycérol-3 Phosphate Oxydase p-4 Aminophénazone (GPO-PAP) pour les triglycérides. Les paramètres contenus dans la bande magnétique de la bandelette, étaient raccordés sur la méthode en Chromatographie sur Gaz/Spectrométrie de Masse (GC-MS). Les échantillons de contrôle suivants ont été utilisés : Reflotron Check, Reflotron Precinorm U et Reflotron précinorm HDL. Les échantillons ont été conservés à $-20{ }^{\circ} \mathrm{C}$ pendant 3 mois, durée garantissant l'intégrité des analytes.

\section{Critères de diagnostic}

La prévalence du pré-diabète a été déterminée suivant les critères de l'OMS (GLY $\geq 6,1 \mathrm{mmol} / 1$ et $<7,0 \mathrm{mmol} / \mathrm{l}$ ) et d'ADA (GLY $\geq 5,6 \mathrm{mmol} / \mathrm{l}$ et $<7,0 \mathrm{mmol} / \mathrm{l})$ (William, 2005; ADA, 2018) auprès des sujets non connus diabétiques. Le critère de glycémie $\geq 7,0 \mathrm{mmol} / 1$ a été utilisé pour évaluer la prévalence du diabète. Les critères de dyslipidémie utilisés étaient : $\mathrm{TG} \geq 1,7$ $\mathrm{mmol} / \mathrm{l}(150 \mathrm{mg} / \mathrm{dl}), \mathrm{CT} \geq 5,2 \mathrm{mmol} / \mathrm{l}(200$ $\mathrm{mg} / \mathrm{dl}$ ) et le Non-HDL-Cholestérol (CT HDL-C) > 4,1 mmol/l $(160 \mathrm{mg} / \mathrm{dl})$. Les critères de consensus de la FID ont été utilisés pour identifier les patients qui auraient un SM : Tour de taille (TT) $\geq 94 \mathrm{~cm}$ pour les hommes $(\mathrm{H})$; TT $\geq 80 \mathrm{~cm}$ pour les femmes (F) ; HDL C < 0,40 (H) ; HDL C < 0,50 (F) ; $\mathrm{TA} \geq 130 / 85 ;$ GLY $\geq 1,00 ; \mathrm{TG} \geq 1,50$ (Alberti et Zimmet, 2007). Pour évaluer un risque élevé de pathologies cardiovasculaires le critère Non-HDL-C > 160 $\mathrm{mg} / \mathrm{dl}$ a été utilisé (Jellinger et al., 2017, Whelton et Carey, 2018). Les critères anthropométriques pour l'Indice de Masse Corporelle (IMC) en poids $(\mathrm{Kg}) /$ taille $(\mathrm{m})^{2}$ étaient ceux de Centers for Disease Control and Prevention (CDC) avec 4 classes: maigreur $<18,5$; normal entre 18,5-24,99; surpoids $\geq 25$; obésité $\geq 30) \quad(C D C, 2007$; Jellinger et al., 2017).

\section{Aspect éthique}

Cette enquête rentre dans le cadre des prérogatives du PNLMCV du Ministère de la Santé qui a assuré et garanti la qualité des dispositifs médicaux utilisés. Un consentement libre et éclairé a été recueilli auprès des personnes enquêtées. Tous les enquêtés ont eu un accès libre à leurs données. Les données recueillies ont été traitées et archivées de façon à sauvegarder la confidentialité. Les sujets qui présentaient des anomalies nécessitant un suivi médical ont été référés vers les structures sanitaires de la ville. L'étude a été réalisée en accord avec la déclaration de bioéthique d'Helsinki et conformément aux exigences de la norme ISO 15189 version 2012.

\section{Analyses statistiques}

Les données recueillies comportaient les antécédents en rapport avec le diabète, l'HTA ainsi que les caractéristiques sociodémographiques. Les différentes données ont été saisies dans un fichier EXCEL et analysées par le logiciel SPSS 21 (IBM version 3.4 de 2018, USA). Les résultats ont été exprimés en moyenne \pm écart-type pour les données à distribution normale et en médiane avec les quartiles pour les données non-paramétriques. Les prévalences ont été exprimées en \% avec les intervalles de confiance (IC) à 95\%. Les principaux tests statistiques utilisés étaient : le test de Kolmogorov-Smirnov, le test du Chi-2 et le test exact de Fischer au besoin. Pour les comparaisons, le test t de Student ou Anova ont été utilisés. Le seuil de significativité utilisé était de 5\%. 
RESULTATS

Aspects démographiques et
caractéristiques diverses
Les sujets inclus dans l'étude provenaient majoritairement du quartier Hedranawoè $(46,8 \%, \mathrm{n}=164)$ avec $49,7 \%$ $(\mathrm{n}=174)$ de 8 autres quartiers de la commune de Lomé (Tokoin, Kodjoviakopé, Kégué, Gbossimé, Bè, Agoe, Adidogomé et Adakpamé) et 3,5\% $(n=12)$ d'autres localités du Togo. Ils étaient $232(66,3 \%)$ femmes versus $33,7 \%(n=118)$ d'hommes, soit deux femmes pour un homme. Ils étaient âgés de 18 à 90 ans avec un âge moyen de 44,5 $\pm 12,6$ ans. Les commerçants étaient prédominants, soit $45,4 \% \quad(n=159)$ dans la population d'étude.

Prévalence du pré-diabète, du diabète, du surpoids, de l'obésité et du syndrome métabolique

L'OMS et ADA ont établi des critères d'identification des sujets en situation de prédiabète. La prévalence du pré-diabète chez les sujets qui ne se connaissaient pas diabétique $(\mathrm{n}=318)$ est détaillée dans le Tableau 1.

Il ressort de ce tableau que le critère ADA a permis de déclarer un nombre plus élevé de sujets pré-diabètiques avec une glycémie moyenne plus proche du seuil de pré-diabète de l'OMS.

La prévalence du diabète au sein de la population d'étude était de 7,1\% ; IC 10,217,5 $(n=25)$. Celle des diabétiques non dépistés, des sujets en surpoids et des sujets obèses sont présentés dans le Tableau 2.

$\mathrm{Ce}$ tableau évoque également la prévalence du SM chez 232 femmes et 118 hommes. Il ressort de ce tableau que la prévalence des diabétiques non dépistés était plus de 5 fois celle des diabétiques, et que la prévalence du SM était plus élevée chez les femmes.

\section{Prévalence du pré-diabète et du diabète} chez les sujets en surpoids ou obèses

Ceux d'entre les sujets d'étude en surpoids ou obèses et non connus diabétiques, étaient au nombre de 142 sujets. La prévalence du pré-diabète au sein de cette sous-population était de :

$$
\begin{aligned}
& -\quad 2,8 \% ; \text { IC } 0,6-5,7(\mathrm{n}=4) \text { selon les } \\
& \text { critères de l'OMS } \\
& \text { - } 13,4 \% ; \text { IC } 8,0-19,4(\mathrm{n}=19) \text { selon } \\
& \quad \text { les critères d'ADA }
\end{aligned}
$$

Le Tableau 3 présente les prévalences du pré-diabète et du diabète distinctement chez les sujets obèses et en surpoids.

Ces détails indiquent que la prévalence du pré-diabète et du diabète étaient plus élevées chez les sujets obèses.

\section{Profil lipidique des pré-diabétiques et diabétiques}

Le diabète et le pré-diabète peuvent entraîner des dyslipidémies. Le Tableau 4 présente en détails leurs prévalences.

Il ressort de ce tableau que les dyslipidémies étaient plus importantes chez les diabétiques.

Tableau 1 : Prévalence du pré-diabète dans la population générale.

\begin{tabular}{cccc}
\hline Critères & $\mathrm{n}(\%)$ & Prévalence & $\begin{array}{c}\text { Glycémie } \\
\text { Moyenne } \pm \text { Ecart-type } \\
\mathrm{mmol} / \mathrm{l}(\mathrm{mg} / \mathrm{dl})\end{array}$ \\
\hline $\begin{array}{c}\text { Pré-diabétiques } \\
\text { selon OMS* }\end{array}$ & $2,5 \%(\mathrm{n}=8)$ & $0,9-4,5$ & $6,5 \pm 0,2 \mathrm{mmol} / \mathrm{l}(116,4 \pm 3,9 \mathrm{mg} / \mathrm{dl})$ \\
$\begin{array}{c}\text { Pré-diabétiques } \\
\text { selon ADA* }\end{array}$ & $13,2 \%(\mathrm{n}=42)$ & $9,6-17,2$ & $5,9 \pm 0,3 \mathrm{mmol} / \mathrm{l}(106,3 \pm 5,8 \mathrm{mg} / \mathrm{dl})$ \\
\hline
\end{tabular}

* OMS : GLY $\geq 6,1 \mathrm{mmol} / 1(110 \mathrm{mg} / \mathrm{dl})$ et $<7,0 \mathrm{mmol} / \mathrm{l}(126 \mathrm{mg} / \mathrm{dl}) / \mathrm{ADA}: \mathrm{GLY} \geq 5,6 \mathrm{mmol} / \mathrm{l}(100 \mathrm{mg} / \mathrm{dl})$ et $<7,0$ $\mathrm{mmol} / \mathrm{l}(126 \mathrm{mg} / \mathrm{dl})$. IC : Intervalle de confiance à $95 \%$. 
Tableau 2 : Prévalence du diabète, du surpoids, de l'obésité et du syndrome métabolique (SM).

\begin{tabular}{ccccc}
\hline \multirow{2}{*}{ Prévalences } & \multicolumn{2}{c}{ Population initiale } & \multicolumn{2}{c}{ Population IMC (n=294)* } \\
& $\%(\mathrm{n})$ & IC & $\%(\mathrm{n})$ & IC \\
\hline Diabétiques & $7,1(25)$ & $2,5-16,2$ & - & - \\
$\begin{array}{c}\text { Diabétiques non } \\
\text { dépistés }\end{array}$ & $40,0(10)$ & $20,8-58,9$ & - & - \\
Surpoids & - & - & $30,3(89)$ & $25,9-34,7$ \\
Obésité & - & - & $24,5(72)$ & $19,2-29,9$ \\
SM (F) & $1,3(3)$ & $0,0-3,0$ & - & - \\
SM (H) & $0,8(1)$ & $0,0-2,6$ & - & - \\
\hline
\end{tabular}

IC : Intervalle de confiance. *il s'agit des sujets donc les données pour IMC étaient disponibles.

Tableau 3 : Prévalence du pré-diabète et du diabète chez les sujets en surpoids et obèses.

\begin{tabular}{cccccc}
\hline Critères & \multicolumn{2}{c}{ Sujets en surpoids $(\mathbf{n = 8 4})$} & \multicolumn{2}{l}{ Sujets obèses $(\mathbf{n = 5 8})$} & p \\
& $\%(\mathrm{n})$ & $\mathrm{IC}$ & $\%(\mathrm{n})$ & $\mathrm{IC}$ & \\
\hline $\begin{array}{c}\text { Pré- } \\
\text { diabétiques } \\
\text { selon OMS }\end{array}$ & $1,2(1)$ & $0,0-4,2$ & $5,2(3)$ & $0,0-11,2$ & 0,463 \\
$\begin{array}{c}\text { Pré- } \\
\text { diabétiques } \\
\text { selon ADA }\end{array}$ & $13,1(11)$ & $6,5-20,0$ & $13,8(8)$ & $5,3-24,7$ & 0,811 \\
\begin{tabular}{c} 
Diabétiques \\
\hline
\end{tabular} & $2,4(2)$ & $0,0-4,2$ & $6,9(4)$ & $2,5-16,2$ & 0,262 \\
\hline
\end{tabular}

IC : intervalle de confiance.

Tableau 4 : Prévalence des dyslipidémies chez les pré-diabétiques et les diabétiques.

\begin{tabular}{|c|c|c|c|c|}
\hline $\begin{array}{c}\text { Critères de } \\
\text { dyslipidémies }\end{array}$ & $\begin{array}{c}\text { Pré-diabète } \\
\text { OMS (n=8) } \\
\%(n) \\
\text { IC }\end{array}$ & $\begin{array}{c}\text { Pré-diabète } \\
\text { ADA }(\mathbf{n}=\mathbf{4 2}) \\
\%(n) \\
\text { IC }\end{array}$ & $\begin{array}{c}\begin{array}{c}\text { Diabète } \\
(\mathbf{n}=\mathbf{2 5})\end{array} \\
\%(n) \\
\text { IC }\end{array}$ & $\mathbf{p}$ \\
\hline $\begin{array}{l}\mathrm{CT} \geq 5,2 \\
\mathrm{mmol} / \mathrm{l}(200 \\
\mathrm{mg} / \mathrm{dl})\end{array}$ & $\begin{array}{c}50,0 \%(n=4) \\
12,2-83,3\end{array}$ & $\begin{array}{c}40,5 \%(n=17) \\
24,5-54,9\end{array}$ & $\begin{array}{c}60,0 \%(\mathrm{n}=15) \\
38,6-77,8\end{array}$ & 0,489 \\
\hline $\begin{array}{l}\text { Non-HDL-C > } \\
4,1 \mathrm{mmol} / \mathrm{l}(160 \\
\mathrm{mg} / \mathrm{dl})\end{array}$ & $0 \%$ & $\begin{array}{c}26,2 \%(n=11) \\
13,2-40,6\end{array}$ & $\begin{array}{c}56,0 \%(\mathrm{n}=14) \\
31,2-76,1\end{array}$ & 0,541 \\
\hline $\begin{array}{l}\mathrm{TG} \geq 1,7 \\
\mathrm{mmol} / \mathrm{l}(150 \\
\mathrm{mg} / \mathrm{dl})\end{array}$ & $\begin{array}{c}12,5 \%(\mathrm{n}=1) \\
0,0-40,0\end{array}$ & $\begin{array}{c}21,4 \%(\mathrm{n}=9) \\
8,5-34,2\end{array}$ & $\begin{array}{c}32,0 \%(\mathrm{n}=8) \\
13,5-51,9\end{array}$ & 0,012 \\
\hline
\end{tabular}




\section{DISCUSSION}

Les prévalences du pré-diabète, du syndrome métabolique et du diabète ont été établies dans une population qui fréquente un marché public, situé dans la région sanitaire de Lomé commune. Les résultats ont montré que la prévalence du pré-diabète dans la population d'étude était de $13,2 \%$ selon les critères d'ADA. La prévalence du diabète était de $7,1 \%$ avec une prévalence des diabétiques non préalablement diagnostiqués à $40 \%$. Ceci confirme le résultat de la Fédération Internationale du Diabète (FID) au sujet de l'Afrique où $70 \%$ de personnes ignore être diabétiques (Suvi, 2017). Celle du SM chez les femmes était plus élevée $(1,3 \%)$. La prévalence du pré-diabète et du diabète chez les sujets obèses, selon les critères de l'OMS était respectivement de 5,2\% et de $6,9 \%$. La prévalence des dyslipidémies était plus élevée chez les diabétiques : 60,0\% pour $\mathrm{CT} \geq 5,2 \mathrm{mmol} / \mathrm{l} ; 56,0 \%$ pour Non-HDL-C $>4,1 \mathrm{mmol} / \mathrm{l}$ et $32,0 \%$ pour $\mathrm{TG} \geq 1,7 \mathrm{mmol} / \mathrm{l}$. $\mathrm{Au}$ Burkina-Faso, une étude réalisée par Koévi et al. (2014) chez 574 diabétiques a montré une corrélation positive en l'artériopathie de ces diabétiques et la dyslipidémie. Il était donc important de déterminer la prévalence des dyslipidémies chez les diabétiques et pré-diabétiques de la présente étude. Les résultats de prévalence du pré-diabète et du diabète ont été obtenus à partir des dosages spectrophotométriques avec des étalons raccordés aux données internationales. Cela atteste de la comparabilité internationale des résultats de cette étude (Supak, 2011). Il s'est agi également d'une mise à jour des données de prévalence du diabète et de la première détermination de la prévalence du pré-diabète auprès d'une population urbaine d'un marché public, car $46,8 \%$ des sujets enquêtés venaient du quartier Hédranawoè où se situe le marché. Et le fait que $49,7 \%$ habitaient dans 8 autres quartiers environnant de ce marché, au sein de la commune de Lomé, atteste que les sujets d'étude étaient urbains. La prévalence du pré-diabète a été établie sur 318 sujets de la population d'étude qui n'étaient pas connus diabétiques pour éviter des biais liés à des patients sous traitement avec des valeurs dans les fourchettes de prédiabète. L'utilisation des Non-HDL-C dans cette étude a permis de s'affranchir de la formule de Friedewald pour le LDL-C qui comporte des limites pour les sujets ayant une $\mathrm{TG} \geq 4,6 \mathrm{mmol} / \mathrm{l}(400 \mathrm{mg} / \mathrm{dl})$. En effet le LDL-C par Friedewald devient non fiable pour les valeurs de TG entre $2,3 \mathrm{mmol} / \mathrm{l}$ ( 200 $\mathrm{mg} / \mathrm{dl})$ et $4,6 \mathrm{mmol} / \mathrm{l}(400 \mathrm{mg} / \mathrm{dl})$.

Les résultats obtenus paraissent concordants avec ceux de l'enquête STEPS de l'OMS au Togo en 2010, dans laquelle la prévalence de l'hyperglycémie diabétique (glycémie capillaire $\geq 110 \mathrm{mg} / \mathrm{dl} \quad(6,1$ $\mathrm{mmol} / \mathrm{l})$ ) était de $2,5 \%$ en milieu urbain (Agoudavi et al., 2012). En utilisant le même critère de l'OMS pour définir les sujets prédiabétiques, au marché de Hédzranawoè, notre étude a trouvé une prévalence identique de $2,5 \%$. En effet, la prévalence de $2,5 \%$ dans cette étude ne concernait que les prédiabétiques et la prévalence des diabétiques a été déterminée séparément comme recommandé par l'association américaine du diabète, ADA (2015). Au Nigéria, une étude similaire de Nwose et al. (2013), dans une population urbaine âgée de 18 à 60 ans, a déterminé une prévalence de pré-diabète de $6,0 \%$ selon les critères de l'OMS, chez les femmes et de $3 \%$ chez les hommes (Nwose et al., 2015). Suivant les critères d'ADA, la prévalence du pré-diabète dans cette étude était inférieure à celle de $18,8 \%$; trouvée par Akter et al. (2011) chez des sujets âgés de plus de 35 ans, en milieu urbain au Bengladesh, un pays asiatique à ressources limitées comme le Togo (Akter et al., 2014). Cela peut s'expliquer par le fait que les sujets de notre étude étaient plus jeunes car recrutés à partir de 18 ans. La glycémie moyenne des pré-diabétiques diagnostiqués selon le critère de glycémie $\geq 5,6 \mathrm{mmol} / \mathrm{l} \quad(100 \mathrm{mg} / \mathrm{dl})$ d'ADA, était de 5,9 $\pm 0,3 \mathrm{mmol} / \mathrm{l}(106,3 \pm 5,8$ $\mathrm{mg} / \mathrm{dl})$. Cette valeur était plus proche du seuil de $6,1 \mathrm{mmol} / \mathrm{l}(110 \mathrm{mg} / \mathrm{dl})$ de l'OMS pour définir les sujets en état de pré-diabète. En effet, avec l'application de l'écart-type, cette 
moyenne se situe entre 5,6 et $6,2 \mathrm{mmol} / \mathrm{l}$, une fourchette qui couvre le seuil de $6,1 \mathrm{mmol} / \mathrm{l}$ de l'OMS. Nous estimons donc que l'utilisation du seuil de $5,6 \mathrm{mmol} / 1$ (100 $\mathrm{mg} / \mathrm{dl})$ d'ADA serait alors plus pertinente dans de pareilles études et en cohérence avec les critères de consensus mondial de la FID concernant le SM établi en 2006 (Alberti et Zimmet, 2007). La prévalence du SM, dans notre étude était similaire à celle trouvée, au cours d'une étude faite sur une population urbaine âgée de 24 à 74 ans au Cameroun en 2007, en utilisant les critères de la FID. Cette étude avait trouvé $1,5 \%$ chez les femmes et $1,2 \%$ chez les hommes sur une population de 1553 sujets dont 887 femmes et 666 hommes avec un ratio de 1,3 contre un ratio de 1,9 en faveur des femmes, dans la présente étude (Ayeska et al., 2014). En 2010 en Ethiopie, une étude incluant 1935 sujets dont 1171 hommes et 764 femmes avec un ratio de 1,5 en faveur des hommes, en utilisant les mêmes critères de la FID, a trouvé une prévalence plus élevée du SM de 14,0\% chez les hommes et de $24,0 \%$ chez les femmes (Tran et al., 2011). Les résultats obtenus au Cameroun étaient similaires aux résultats de la présente étude avec une prévalence plus élevée chez les femmes comme c'était le cas en Ethiopie aussi. Une étude réalisée au Benin sur 270 hypertendus, mais avec le critère de la NCEP pour déterminer la prévalence du SM a également trouvé que cette prévalence était significativement plus élevée chez les femmes (Assoumanou et al., 2012). Cette prévalence plus élevée chez les femmes ne serait pas liée au fait que les populations d'étude étaient constituées de plus de femmes. D'autres études ont montré que cette prévalence élevée chez les femmes, était liée à la présence de graisse souscutanée abdominale souvent importante chez les femmes africaines. Cette graisse souscutanée serait corrélée à des déterminants cardio-métaboliques comme l'insulinorésistance (IR) dont le ratio CT/HDL-C serait prédicteur (Sossa et al., 2015 ; Gradidge et Crowther, 2017). Cependant il est à noter que la prévalence du SM dans l'étude de Tran et al en Ethiopie était 18,5 fois plus élevée que celle des femmes dans l'étude présente. Le critère de la FID a proposé d'utiliser des normes européennes de TT pour les tous africains. Ces critères de la FID pour le diagnostic du SM ont également permis d'identifier des sujets en état de pré-diabète avec des dyslipidémies. Il serait nécessaire que des normes de TT soient établies pour des sujets de l'Afrique Sub-Saharienne; avec une évaluation de la valeur prédictive de l'IR grâce à des outils simples comme le ratio CT/HDL-C.

Dans la présente étude, les prévalences des dyslipidémies étaient de $21,4 \%$ pour les TG et de $40,5 \%$ pour la CT chez les pré-diabétiques identifiés avec le critère ADA. Bishwajit et al. (2018) au Bengladesh en 2009, chez des sujets âgés d'au moins 20 ans ont trouvé que $13,2 \%$ des pré-diabétiques avaient une $\mathrm{CT} \geq 5,2 \mathrm{mmol} / 1$ contre $40,5 \%$ dans la présente étude. Et $42,1 \%$ qui avaient une $\mathrm{TG} \geq 1,7 \mathrm{mmol} / \mathrm{l}$ contre $21,4 \%$ dans cette étude. En effet, une prévalence plus élevée de triglycéridémie comme dans cette étude pourrait provenir, entre autres, d'une consommation excessive de fructose contenu en quantité plus importante dans les aliments suivants : édulcorant tagatose dans les boissons non alcoolisées, jus de fruits de commerces (pomme, mangue, orange, goyave), barres de céréales, chewing-gums, confitures et tout aliment transformé avec une étiquette mentionnant la présence de lévulose et sucre inverti. Egalement un mode d'alimentation caractérisé par la consommation des aliments à index glycémique (IG) supérieur à 70 (pain blanc sucré, pain de mie, corn flakes, riz blanc à cuisson rapide, frites de pomme de terre et bonbons) entraîne un excès d'apport glucidique qui favorise la synthèse d'acides gras contribuant à une hypertriglycéridémie. Ces aliments sont souvent très présents sur les places du marché. Une investigation des habitudes alimentaires permettrait de confirmer le type de métabolisme en accord avec les résultats obtenus. (Houlbert et al., 2008 ; Schlienger, 2014). La prévalence du 
pré-diabète et du diabète était plus élevée chez les sujets obèses comparativement à ceux en surpoids. Il a été établi que l'obésité est un facteur prédictif du pré-diabète. La prévalence du pré-diabète peut être attribuée non seulement au degré ou à la sévérité de l'obésité, mais aussi à la distribution de la graisse au niveau du corps (Gaw et al. 2004 ; Compeán-Ortiz et al., 2017). Osei et Gaillard (2019) aux USA ont établi que, les américaines noires obèses d'origine africaine, avaient une prévalence plus élevée du pré-diabète et du diabète de type 2 . Qu'en est-t-il des femmes noires en Afrique Subsaharienne et des sujets jeunes et non obèses. Ces études récentes montrent la nécessité de s'intéresser spécifiquement à ces types de sujets (Mainous et al., 2016). D'autres études dans des marchés publics de Lomé et des autres villes du Togo, en ciblant les jours d'affluences des marchés pour un recrutement plus important de sujets seraient nécessaires.

\section{Conclusion}

Cette étude a montré que la prévalence du pré-diabète et du diabète était en augmentation dans la population urbaine fréquentant le marché de Hédzranawoè. Ces prévalences étaient plus élevées chez les sujets obèses. Les sujets diabétiques avaient une prévalence élevée de dyslipidémies, et donc un risque élevé de maladies cardiovasculaires. La prévalence $\mathrm{du}$ syndrome métabolique était plus élevée chez les femmes. Elle mérite d'être réévaluer en utilisant des critères de tour de taille établis pour des africains au Sud du Sahara.

\section{CONFLIT D'INTERETS}

Les auteurs déclarent n'avoir aucun conflit d'intérêts concernant les données publiées dans cet article.

\section{CONTRIBUTIONS DES AUTEURS}

KCK a Participé au recueil des données, à la validation des examens biochimiques, au suivi des analyses statistiques et à la rédaction de l'article.
SP a Participé au recueil des données cliniques et de la rédaction de l'article.

LK a Participé aux installations logistiques et au recueil des données cliniques.

CL a Participé aux évaluations biochimiques et à la relecture.

FD est le Responsable du Programme National de Lutte contre les Maladies CardioVasculaire du Ministère de la Santé. Il est l'initiateur de l'étude et a participé à sa validation finale.

\section{REMERCIEMENTS}

A tous les infirmiers, techniciens de laboratoires et autres médecins du CHU Campus ayant participé à cette étude.

\section{REFERENCES}

Agoudavi K, Adjoh KS, Afanvi K, Agbétsiafa K, Amédegnato $\mathrm{D}$, Baba AB, Dogbé KS Tamékloe T. 2010. Rapport final de l'enquête STEPS Togo en 2010: Service des maladies nontransmissibles de la division d'épidémiologie. Ministère de la sante $\mathrm{du}$ togo. [https://www.who.int/ncds/surveillance /steps/2010STEPS_Report_Togo_FR.p df].

Akter S, Rahman M, Krull-Abe S, Sultana P. 2014. Prevalence of diabetes and prediabetes and their risk factors among Bangladeshi adults: a nationwide survey. Bull. World Health Organ., 92: 204-213.

DOI: https://doi.org/10.2471/BLT.13.128371

Alberti G, Zimmet P. 2007. Consensus worldwide definition of the metabolic syndrome. International Diabetes Federation (IDF) Communications, Brussels.

American Diabetes Association. 2015. Classification and diagnosis of diabetes: standards of Medical Care in Diabetes. Diabetes Care, 38(1): S8-S16. DOI: https://doi.org/10.2337/dc15-S005

American Diabetes Association. 2018. Glycemic targets: standards of medical 
care in diabetes. Diabetes Care, 41(1):

S55-S64.

DOI:

https://doi.org/10.2337/dc18-S006

Assoumanou MG, Dovonou AC, Ngome

MR, Akpona SA. 2012. Prévalence du syndrome métabolique chez les sujets hypertendus adultes dans les formations sanitaires de Parakou (Bénin). Int. J. Biol. Chem. Sci., 6(4): 1419-1427. DOI: http://dx.doi.org/10.4314/ijbcs.v6i4.2

Motola AA, Mbanya JC, Kaushik LR. 2009. Metabolic syndrome in sub-saharan Africa. Ethn. Dis., 19 (2): S2.8-S2.10

Bhowmik B, Siddiquee T, Mujumder A, Afsana F, Ahmed T, Mdala IA, Moreira NCV, Khan AKA, Hussain A, Holmboe-Ottesen G, Omsland TK. 2018. Serum lipid profile and its association with diabetes and prediabetes in a rural Bangladeshi population. Int. J. Environ. Res. Public Health,

15:

2-12.

DOI: https://doi.org/10.3390/ijerph150 91944

CDC (Centers for Disease Control and Prevention). Defining Adult Overweight and Obesity. CDC, Atlanta. [https://www.cdc.gov/obesity/adult/def ining.html]. (consulté le 20 mai 2020).

Compeán-Ortiz LG, Trujillo-Olivera LE, Valles-Medina AM, ReséndizGonzález E, Beatriz García-Solano B, Pérez BDA.2017. Obesity, physical activity and prediabetes in adult children of people with diabetes. Rev. Latino-Am. Enfermagem., 25: e2981-7. DOI: https://doi.org/10.1590/15188345.2102.2981

Gaw A, Murphy M, Cowan R. 2004. Métabolisme du glucose et diabète sucré; diagnostic et surveillance du diabète. In Biochimie Clinique, Gaw A, Murphy $J M$, Cowan $A R$, O'reilly St JD, Stewart JM, Sheperd J (ed). ElsevierMasson: Issy-les-Moulineaux; 58-59.

Gradidge JL, Crowther NJ. 2017. Metabolic syndrome in black south african women. Ethn. Dis., 27(2): 189-200.
DOI: https://doi.org/10.18865/ed.27.2. 189

Houlbert A, Molénat V, Nérin E, Périault A, Soleille C, Souccar T, Tremblais P. 2008. La meilleure façon de manger. Le premier guide alimentaire basé sur la science (ed). Marabout Santé : Vergèze.

Jellinger P, Handelsman Y, Rosenblit P, Bloomgarden Z, Fonseca V, Garber A, Grunberger G, Guerin C, Bell D, Mechanick J, Pessah-Pollack R, Wyne $\mathrm{K}$, Smith $\mathrm{D}$, Brinton E, Fazio S, Davidson M. 2017. American association of clinical endocrinologists and american college of endocrinology guidelines for management of dyslipidemia and prevention of cardiovascular disease. Endocrine Practice, 23(2): 1-87. DOI: https://doi.org/10.4158/EP171764.APP GL

Koevi KKA, Millogo V, Ouedraogo M, Ouedraogo GA. 2014. Diagnostic des causes de complication du diabète et des méthodes de prévention à BoboDioulasso, au Burkina Faso. Int. J. Biol. Chem. Sci., 8(6): 2709-2720. DOI: http://dx.doi.org/10.4314/ijbcs.v8i6.30

Mainous AG, Tanner RJ, Jo A, Anton SD. 2016. Prevalence of prediabetes and abdominal obesity among healthyweight adults: 18 - year trend. Ann. Fam. Med., 14: 304-310 DOI: https://doi.org/10.1370/afm.1946

National Institutes of Health. 2001. Expert panel on detection, evaluation, and treatment of high blood cholesterol in adults: executive summary of the third report of the national cholesterol education program (NCEP). JAMA., 285:

2486.

DOI: https://doi.org/10.1001/jama.285. 19.2486

Nwose EU, Oguoma VM, Bwititi PT, Richards RS. 2015. Metabolic Syndrome and Prediabetes in Ndokwa Community of Nigeria: preliminary Study. North Am. J. Med. Sci., 7 (2): 53- 
58. DOI: https://doi.org/10.4103/19472714.152079

Okafor CI. 2012. The metabolic syndrome in Africa: current trends. Indian $J$. Endocrinol. Metab., 16 (1): 56-66. DOI: https://doi.org/10.4103/22308210.91191

Osei K, Gaillard T. 2019. Pathogenic mechanisms of prediabetes in obese vs very obese African American women: implications for diabetes prevention. Journal of the National Medical Association, 111(1): 76-82. DOI: https://doi.org/10.1016/j.jnma.20 18.06.004

Schlienger J-L. 2014. Syndrome métabolique. In Nutrition Clinique Pratique: Chez L'Adulte et l'Enfant, Schlienger J-L (ed). Elsevier-Masson: Issy-les-Moulineaux; 161-163

Shen J, Kondal D, Rubinstein A, Irazola V, Gutierrez L, Miranda JJ, Bernabé-Ortiz A, Lazo-Porras M, Levitt N, Steyn K, Bobrow K, Ali MK, Prabhakaran D, Tanon N. 2016. A multiethnic study of pre-diabetes and diabetes in low and medium income countries. Global Heart, 11(1): 61-70. DOI: https://doi.org/10.1016/j.gheart.2015.1 2.015

Sossa C, Agueh V, Saizonou J, Moussiliou PN, Kpozehouen A, Makoutode M,
Delisle H. 2015. Prédiction de l'insulino-résistance par les ratios de lipoprotéines chez les adultes béninois. Int. J. Biol. Chem. Sci., 9(3): 13191329.

DOI: http://dx.doi.org/10.4314/ijbcs.v9i3.17

Supak S. 2011. Validation of methods performance for routine biochemistry. Biochemia Medical, 21(2): 182-90. DOI: https://doi.org/10.11613/BM.2011.028

Suvi K, Joao de Roche F, Yadi H, Belama M. 2017. FID des Atlas du Diabète (8 ${ }^{\text {ème }}$ Edn). IDF Communications: Brussels.

Tran A, Gelaye B, Girma B, Lemma S, Berhane Y, Bekele T, Khali A, Williams MA. 2011. Prevalence of metabolic syndrome among working adults in Ethiopia. Int. J. Hypertension, 193719: $1-8$. DOI: https://doi.org/10.4061/2011/193719

Whelton PK, Carey RM. 2018. Guideline for the prevention, detection, evaluation and Management of High blood pressure in Adults. JACC., 71(19): e127-248.

William BS. 2005. Pathologies du métabolisme glucidique. In Biochimie Médicale, Physiopathologie et Diagnostic, Marshall JW, Bangert KS (ed). Elsevier-Masson: Issy-lesMoulineaux; 331-341. 